\title{
Utilidade do benzoato de estradiol após a suplementação com gestágeno na sincronização de cios de novilhas de corte*
}

\author{
The effect of estradiol benzoate after progestagen supplementation for estrus \\ synchronization in beef heifers \\ Guilherme Vogg ${ }^{1}$, Carlos J osé Hoff de Souza ${ }^{2}$, Carlos Miguel Jaume ${ }^{3}$ \\ \& José Carlos Ferrugem Moraes ${ }^{3}$
}

\begin{abstract}
RESUMO
Existem evidências de que a associação de benzoato de estradiol (ODB) após a suplementação com progesterona concentra a manifestação de cios em sistemas de sincronização de cios para bovinos. O objetivo deste experimento foi de verificar o efeito do uso de diferentes dosagens de ODB injetadas 24 horas após a retirada do progestágeno, em novilhas de 14 e 24 meses de idade. Foram utilizadas 376 novilhas que receberam esponjas vaginais durante 7 dias e na colocação dos pessários 5,0 mg de ODB i.m.. Na retirada das esponjas foram distribuídas em três tratamentos: 0,0 ODB, que não receberam o estrógeno; $0,5 \mathrm{ODB}$, com 0,5 mg e 1,0 ODB, com 1,0 mg de estrógeno i.m. 24 h após a retirada do pessário. As variáveis medidas foram: cio (presença ou não), $2^{\circ}$ cio (taxa de segundo serviço) e prenhez, ao final da temporada reprodutiva. Nas novilhas de 24 meses que receberam ODB foi observada maior frequiência de cios na propriedade A, em contraste, na propriedade B a taxa de $2^{\circ}$ cio foi superior. No geral foi observada uma taxa de cio de $84 \%, 96 \%$ e $96 \%$ e de $2^{\circ}$ cio de $19 \%, 35 \%$ e $45 \%$, respectivamente para $0,0 \mathrm{ODB}, 0,5 \mathrm{ODB}$ e 1,0 ODB. As novilhas de 14 meses tratadas com ODB manifestam um maior percentual de cios, sem modificação significativa na taxa de retorno. A associação de $0,5 \mathrm{mg}$ de ODB à suplementação com o progestágeno promove maior incidência de cio em novilhas de 24 meses, porém, com redução da fertilidade em algumas situações. A utilidade da associação com esteróides é maior em novilhas de 14 meses, nas quais as taxas de concepção foram semelhantes as que não receberam o estrógeno.
\end{abstract}

Descritores: sincronização de cios, progestágenos, bovinos de corte.

\begin{abstract}
There are evidences that the association of estradiol benzoate (ODB) to progesterone supplementation concentrates estrus behavior in synchronization systems for cattle. The aim of this study was to investigate the effect of different concentrations of ODB 24 after progestagen pessary withdrawal in heifers aging 14 and 24 months, on estrus behavior after synchronization. We used 376 heifers that were treated with pessaries for 7 days and $5 \mathrm{mg}$ of ODB at pessary insertion and allocated to one of the following treatments: 0,0 ODB, no further treatments; $0,5 \mathrm{ODB}$, injection of $0,5 \mathrm{mg}$ ODB i.m.; 1,0 ODB, injection of 1,0 mg ODB i.m. $24 \mathrm{~h}$ after pessary withdrawal. The variables investigated were the presence of estrus after synchronization (cio), rate of second service ( $2^{\circ}$ cio) and gestation rate at the end of the reproductive season (prenhez). In the 24 months old heifers ODB supplementation increased in the incidence of estrus at farm A, in contrast, at farm B the ODB supplementation increased the incidence of return to service. The overall first estrus rate was $84 \%, 96 \% \mathrm{e}$ $96 \%$ and second estrus rate was $19 \%, 35 \%$ e $45 \%$, for 0,0 ODB, 0,5 ODB e 1,0 ODB, respectively. The 14 month old heifers treated with $0,5 \mathrm{mg}$ ODB showed a higher incidence of estrus, without changing the return rate. The supplementation with $0,5 \mathrm{mg}$ ODB at pessary withdrawal promoted higher incidence of estrus in the older heifers, but with a fertility reduction in some instances. The utility of ODB supplementation is greater in 14 month old heifers, which show the same conception rate as the non-treated females.
\end{abstract}

Key words: estrus synchronization, progestagen, beef cattle. 


\section{INTRODUÇÃO}

A produtividade de um sistema extensivo de produção de carne bovina está em função da fertilidade das vacas [9]. A sincronização dos cios das novilhas de primeira cria viabiliza uma maior concentração dos partos no terço inicial do período de parição, permitindo que estas tenham mais tempo para restabelecerem suas reservas corporais no pós-parto.

A concentração das parições e a redução das temporadas reprodutivas, podem ser obtidas com diferentes combinações de progestágenos e prostaglandinas [5,13]. No sul do Brasil, Uruguai e Argentina, têm sido desenvolvidos para sincronização de cios em bovinos pessários impregnados com gestágenos [2,12] ou com progesterona natural [3].

Alguns estudos têm indicado que a associação de estrógenos à progesterona/progestágenos seria útil para elevar a taxa de manifestação de cio e de prenhez em novilhas próximas da puberdade, e, também em vacas paridas em anestro [1,4, 10,14]. A associação de $0,5 \mathrm{mg}$ de benzoato de estradiol (ODB) 24 horas após a remoção de implantes vaginais com progesterona, incrementou a taxa de sincronização e de prenhez em novilhas [7]. Em contraste, existem evidências de que a associação de progestágenos com estradiol 24 e 16 horas após a remoção dos implantes reduz a taxa de concepção ao primeiro serviço [15,17]. O uso do ODB 24 h após a remoção dos pessários, reduz o intervalo para a manifestação do cio, a variabilidade no momento do início do cio sem afetar a taxa de prenhez quando aplicado na emergência da onda folicular [11].

O objetivo deste experimento foi de verificar o efeito do uso de diferentes dosagens de ODB injetadas 24 horas após a retirada do progestágeno, em novilhas de 14 e 24 meses de idade.

\section{MATERIAIS E MÉTODOS}

Os ensaios experimentais foram executados em duas propriedades nos municípios de Rio Pardo (Propriedade A) e Cachoeira do Sul (Propriedade B) na região central do Estado do Rio Grande do Sul. O trabalho foi desenvolvido entre os meses de dezembro de 1998 e fevereiro de 1999. Foram utilizadas 376 novilhas de corte, predominantemente da raça Angus, sendo 122 na propriedade A com idade média de 24 meses e 254 na propriedade B, destas, 196 com idade média de 24 meses e 58 com idade média de 14 meses. As novilhas da propriedade A no início do acasalamento apresentavam um peso médio de $261 \pm 2 \mathrm{~kg}$ e as da propriedade $\mathrm{B}$, respectivamente de $282 \pm 3 \mathrm{~kg}$ e $312 \pm 2 \mathrm{~kg}$ para as de 14 e 24 meses de idade.

As novilhas receberam esponjas vaginais impregnadas com $250 \mathrm{mg}$ de acetato de medroxiprogesterona durante 7 dias e na colocação dos pessários 5,0 $\mathrm{mg}$ de benzoato de estradiol i.m.. $\mathrm{Na}$ retirada das esponjas foram distribuídas em três tratamentos: 0,0 ODB, que não receberam o estrógeno; 0,5 ODB, com 0,5 mg de benzoato de estradiol; e, 1,0 ODB, com 1,0 mg de benzoato de estradiol i.m. $24 \mathrm{~h}$ após a retirada do pessário.

Após a retirada dos pessários as novilhas foram controladas diariamente durante 24 dias, sendo de 26/12/98 a 18/01/99 na propriedade A e de 4/12/98 a 30/12/98 na propriedade B. O controle para detecção do estro foi feito duas vezes ao dia, pela manhã das 8:00 h as 9:30 h e pela tarde das 17:00 h as 18:30 h. Os animais detectados em estro foram inseminados 12 horas após a observação do cio. Ao final do período de controle as novilhas permaneceram com touros no percentual de $2 \%$ por 61 dias, totalizando um período de monta de 85 dias. A manifestação de cio e as datas de primeira e segunda inseminação foram anotadas, $o$ que originou as variáveis cio (presença ou não) e taxa de segundo serviço ( $2^{\circ}$ cio). O diagnóstico de gestação com base na palpação retal foi realizado 40 dias após o término da temporada de monta (Prenhez). As variáveis medidas foram analisadas através de Qui-quadrado simples e de heterogeneidade, considerando as fontes de variação, tratamento, propriedade e idade.

\section{RESULTADOS}

Na Tabela 1 são apresentados os resultados da investigação da eficácia da sincronização de cios nas novilhas de 24 meses, incluindo dados das duas propriedades. Pode ser observado um incremento significativo na manifestação de cios na propriedade $\mathrm{A}$, quando o MAP foi associado ao ODB, sem efeito significativo na taxa de retorno à inseminação ( $2^{\circ}$ cio). A taxa efetiva de prenhez foi maior nas novilhas tratadas com $0,5 \mathrm{mg}$ de ODB. Em contraste com esses dados, na propriedade $\mathrm{B}$ a taxa de retorno ao cio foi superior nas novilhas que receberam ODB. A análise combinada das duas propriedades $\mathrm{A}+\mathrm{B}$, revelou maior porcentual de manifestação de cios e de retorno as inseminações quando 
Tabela 1. Incidência de cio e fertilidade de novilhas de 24 meses de duas propriedades, submetidas a sincronização com pessários de acetato de medroxi-progesterona com ou sem injeção de benzoato de estradiol 24 horas após a retirada dos pessários.

\begin{tabular}{|c|c|c|c|c|c|}
\hline \multirow[b]{2}{*}{ Propriedade } & \multirow[b]{2}{*}{ Variável } & \multicolumn{3}{|c|}{ Tratamentos } & \multirow[b]{2}{*}{$\chi^{2} \#$} \\
\hline & & $0,0 \mathrm{mg}$ ODB & $0,5 \mathrm{mg}$ ODB & $1,0 \mathrm{mg}$ ODB & \\
\hline \multirow[t]{3}{*}{ A } & $1^{\circ}$ cio & $31 / 40(78 \%)$ & $39 / 40(98 \%)$ & $41 / 42(98 \%)$ & $13,19^{* *}$ \\
\hline & $2^{\circ}$ cio & $6 / 40(15 \%)$ & $15 / 40(38 \%)$ & $12 / 42(29 \%)$ & 5,21 \\
\hline & Prenhez & $21 / 40(53 \%)$ & $30 / 40(75 \%)$ & $21 / 42(50 \%)$ & $6,34^{*}$ \\
\hline \multirow[t]{3}{*}{ B } & $1^{\circ}$ cio & $57 / 65(88 \%)$ & 63/66 (95\%) & $62 / 65$ (95\%) & 3,91 \\
\hline & $2^{\circ}$ cio & $14 / 65(21 \%)$ & $22 / 66(33 \%)$ & $36 / 65$ (55\%) & $16,52^{* *}$ \\
\hline & Prenhez & $57 / 64$ (89\%) & $57 / 65$ (88\%) & $62 / 65$ (95\%) & 2,60 \\
\hline \multirow[t]{3}{*}{$A+B$} & $1^{\circ}$ cio & $88 / 105(84 \%)$ & $102 / 106(96 \%)$ & $103 / 107(96 \%)$ & $15,01^{\star *}$ \\
\hline & $2^{\circ}$ cio & $20 / 105(19 \%)$ & $37 / 106(35 \%)$ & $48 / 107(45 \%)$ & $16,22^{* *}$ \\
\hline & Prenhez & $78 / 104(75 \%)$ & $85 / 105(83 \%)$ & $83 / 107(78 \%)$ & 1,99 \\
\hline
\end{tabular}

os tratamentos incluíram a associação com ODB. O somatório das frequiências pode ser assumido como real, considerando que o qui-quadrado de heterogeneidade indica que a manifestação do primeiro cio foi semelhante para os tratamentos testados nas duas propriedades $\left(\chi^{2}=2,09 ; 2 \mathrm{GL} ; \mathrm{P}>0,30\right)$, bem como para o percentual de retorno $\left(\chi^{2}=5,50 ; 2 \mathrm{GL} ; \mathrm{P}>0,05\right)$.

No que concerne a taxa de prenhez, embora incluindo o período de monta natural é interessante salientar que as duas propriedades são heterogêneas $\left(\chi^{2}\right.$ het $\left.=6,95 ; 2 \mathrm{GL} ; \mathrm{P}<0,01\right)$, sendo caracterizadas por taxas de gestação bem diferenciadas.

A comparação da eficácia da associação do estrógeno com o MAP em novilhas de 14 e 24 meses de idade foi efetivada na propriedade B. Na Tabela 2 são indicadas as frequiências de $1^{\circ} \mathrm{e} 2^{\circ}$ cios e percentual de prenhez nos dois conjuntos de animais. As novilhas de 14 meses quando tratadas já com $0,5 \mathrm{mg}$ de ODB manifestam um maior percentual de cios, sem modificação na taxa de retorno ( $2^{\circ}$ cio). Nas novilhas de 24 meses, o uso do ODB não modificou de maneira significativa a manifestação de estros, porém, foi observada uma maior percentagem de retorno ao cio naquelas tra- tadas com 1,0 mg de ODB. De uma maneira geral na propriedade $\mathrm{B}$ (análise combinada das duas idades), fica evidente que o uso de benzoato de estradiol associado ao MAP na sincronização de cios proporciona maior taxa de inseminação nas duas doses testadas e maior percentagem de retorno no tratamento $1,0 \mathrm{mg}$ ODB em comparação ao grupo controle não tratado $(0,0$ ODB). O percentual de novilhas gestantes no final da temporada reprodutiva também foi superior nas tratadas com 1,0 mg ODB. Os desvios entre idade e tratamento foram no mesmo sentido, sendo possível analisar os dados de maneira conjunta para a manifestação do $1^{\circ}$ cio $\left(\chi^{2}\right.$ het $\left.=1,76 ; 2 \mathrm{GL} ; \mathrm{P}>0,30\right)$, incidência de novo cio após a inseminação $\left(\chi^{2}\right.$ het $\left.=4,55 ; 2 \mathrm{GL} ; \mathrm{P}>0,05\right) \mathrm{e}$ taxa de prenhez $\left(\chi^{2}\right.$ het $\left.=0,65 ; 2 \mathrm{GL} ; \mathrm{P}>0,05\right)$.

O tempo para manifestação do primeiro cio foi semelhante entre propriedades e idades, assim os dados foram analisados em conjunto. Na Figura 1 pode ser visualizada a distribuição do momento da manifestação dos cios para os três tratamentos testados estratificados em quatro classes de horários (até 24h, até $48 \mathrm{~h}$, até 72 e até 120 h). As frequiências de cios em cada classe foram distintas entre os tratamentos 
Vogg G., Souza C.J.H., J aume C.M. \& Moraes J.C.F. 2004 . Utilidade do benzoato de estradiol após a suplementação com gestágeno na sincronização de cios de novilhas de corte.

Acta Scientiae Veterinariae. 32: 41-46.

$\left(\chi^{2}=34,78 ; 6 \mathrm{GL} ; \mathrm{P}<0,01\right)$. Nas primeiras 24 horas os grupos tratados com ODB, apresentaram $61,3 \%$ e $66,1 \%$, respectivamente para $0,5 \mathrm{mg}$ ODB e $1,0 \mathrm{mg}$ ODB, em contraste com apenas $33 \%$ para o grupo 0,0 ODB (não tratado com o estrógeno).

\section{DISCUSSÃO}

A manifestação de estro é a chave para o sucesso dos programas convencionais de inseminação artificial. Em rebanhos ciclando, cerca de 5\% das vacas manifestam cio por dia, com a utilização de sincronização de cios com acetato de medroxi-

Tabela 2. Incidência de cios e fertilidade de novilhas de 14 e 24 meses, submetidas a sincronização com pessários de acetato de medroxi-progesterona com ou sem injeção de benzoato de estradiol 24 horas após a retirada dos pessários.

\begin{tabular}{|c|c|c|c|c|c|}
\hline \multirow[b]{2}{*}{ Idade } & \multirow[b]{2}{*}{ Variável } & \multicolumn{3}{|c|}{ Tratamentos } & \multirow[b]{2}{*}{$\chi 2 \#$} \\
\hline & & $0,0 \mathrm{mg}$ ODB & $0,5 \mathrm{mg}$ ODB & $1,0 \mathrm{mg}$ ODB & \\
\hline \multirow[t]{3}{*}{14 meses } & $1^{\circ}$ cio & $11 / 20(55 \%)$ & $17 / 19(89 \%)$ & $18 / 19(95 \%)$ & $11,16^{* *}$ \\
\hline & $2^{\circ}$ cio & $4 / 20(20 \%)$ & $6 / 19(32 \%)$ & $9 / 19(47 \%)$ & 3,33 \\
\hline & Prenhez & $14 / 20(70 \%)$ & $14 / 19(74 \%)$ & $18 / 19(95 \%)$ & 4,18 \\
\hline \multirow[t]{3}{*}{24 meses } & $1^{\circ}$ cio & $57 / 65(88 \%)$ & $63 / 66(95 \%)$ & $62 / 65$ (95\%) & 3,92 \\
\hline & $2^{\circ}$ cio & $14 / 65(22 \%)$ & $22 / 66(33 \%)$ & $36 / 65$ (55\%) & $16,52^{* *}$ \\
\hline & Prenhez & $57 / 64(89 \%)$ & $57 / 65$ (88\%) & $62 / 65$ (95\%) & 2,60 \\
\hline \multirow[t]{3}{*}{$14+24$ meses } & $1^{\circ}$ cio & $68 / 85(80 \%)$ & $80 / 85(94 \%)$ & $80 / 84(95 \%)$ & $13,31^{* *}$ \\
\hline & $2^{\circ}$ cio & $18 / 85(21 \%)$ & 28/85(33\%) & $45 / 84(54 \%)$ & $15,29^{* *}$ \\
\hline & Prenhez & $71 / 84(85 \%)$ & $71 / 84(85 \%)$ & $80 / 84(95 \%)$ & $6,13^{*}$ \\
\hline
\end{tabular}

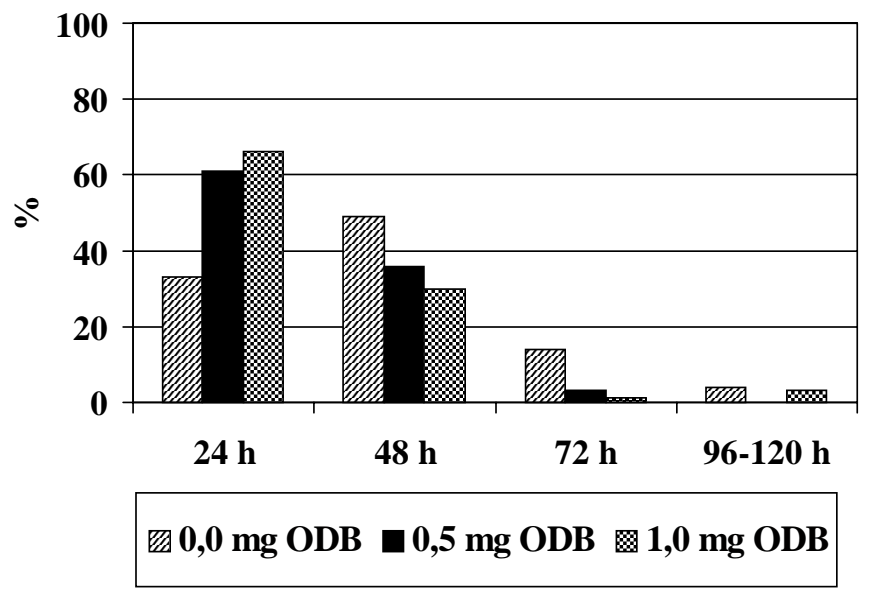

Figura 1. Distribuição do início da manifestação dos cios após a injeção de benzoato de estradiol 24 horas após a remoção dos pessários. progesterona, da remoção do pessário com o gestágeno até $120 \mathrm{~h}$ após, foram identificadas em cio 84\% (78\% na propriedade A e $88 \%$ na propriedade B) das novilhas de 24 meses e $55 \%$ das novilhas de 14 meses. Portanto, é indiscutível a utilidade desses sistemas para a concentração dos partos, notadamente, considerando que a taxa de segundo serviço foram baixas, oscilando de $15 \%$ a $22 \%$ quando o sistema de sincronização de cios não está associado ao ODB. Os dispositivos similares desenvolvidos no Uruguai e Argentina apresentaram eficácia na manifestação de cios inferior, porém, muito provavelmente em decorrência de terem sido testados, respectivamente em vacas leiteiras lactantes e vacas de corte com cria ao pé $[2,3]$.

A associação do gestágeno com o benzoato de estradiol tem sido alvo de inúmeros estudos nas últimas 
décadas, visando associar os possíveis benefícios fisiológicos do estrógeno ao seu baixo custo comercial. No entanto, o uso de $0,5 \mathrm{mg}$ a $1,0 \mathrm{mg}$ de ODB no momento da remoção dos pessários ( $\left.7^{\circ} \mathrm{dia}\right)$ em vacas com cria ao pé [16] e em novilhas entre 16 e 24 h após a suplementação com progesterona $[15,17]$ determinou cerca de 20-25\% de redução na taxa de concepção. Estes resultados não se confirmaram neste ensaio em novilhas, no qual, também foram investigados possíveis efeitos de idade e de propriedade. Entretanto, é possível inferir que $0,5 \mathrm{mg}$ de ODB deve ser a dose máxima, que as novilhas respondem sem afetar a fertilização do oócito cujo folículo está prestes a ovular. Nestas condições, sem uma importante e repetitiva redução na fertilidade, é possível recomendar a associação do ODB para reduzir a variabilidade da manifestação do cio após a suplementação com medroxiprogesterona.

O possível efeito na fertilidade detectado em alguns estudos deve ser decorrente do período de suplementação e da fase da onda folicular em que o ODB é administrado, o que foi recentemente demonstrado [11]. Uma possível explicação para a disparidade dos resultados pode ser a seguinte: quando não foi observado efeito sobre a fertilidade, o ODB foi administrado 24-30 h após a remoção dos dispositivos impregnados com progesterona, que permaneceram na vagina das vacas durante 10 a 12 dias, portanto, com alta probabilidade de ser injetado durante a fase de dominância $[8,18]$. Essa possibilidade foi reiterada por um experimento [1] que investigou a utilidade de apenas 5 dias de permanência do dispositivo vaginal, verificando, nesse caso, menor percentagem de prenhez, em vacas com mais de 28 dias pós-parto.

Os dados da Figura 1 indicam que efetivamente há menor variabilidade no momento de detecção dos cios e possivelmente maior sincronização das ovulações, também pode ser obtida, quando o ODB é associado ao acetato de medroxi-progesterona à semelhança do que foi verificado para a progesterona $[7,10]$.

Um fato inusitado constatado no presente estudo foram as altas taxas de cio verificadas nas novilhas, isso, tendo em conta o baixo efeito luteolítico do ODB, até mesmo na dose de $10 \mathrm{mg}$ em cápsulas [6]. Porém em novilhas próximas a puberdade e/ou retornando do anestro estacional (causado pela restrição alimentar decorrente do inverno anterior) a suplementação com o gestágeno pode ser o fator determinante dessas respostas, até mesmo sem a associação com o ODB [14].

Adicionalmente é interessante mencionar a diferença significativa nos percentuais de prenhez durante toda a temporada reprodutiva, que devem ser decorrentes das condições de criação e/ou qualidade das pastagens e de solo são superiores na propriedade $\mathrm{B}$, tanto que foi possível submeter ao primeiro acasalamento $23 \%$ das novilhas já aos 14 meses, com peso corporal aceitável para esses conjuntos raciais.

\section{CONCLUSÕES}

$\mathrm{A}$ associação de $0,5 \mathrm{mg}$ de $\mathrm{ODB}$ à suplementação com acetato de medroxi-progesterona promove maior taxa de manifestação de cio em novilhas de 24 meses, porém, com menores taxas de concepção ao primeiro serviço em algumas situações peculiares relativas às unidades de produção. A utilidade da associação dos esteróides é maior em novilhas de 14 meses, nas quais as taxas de concepção é normal, o que pode ser decorrente de uma condição de anestro diferenciada daquelas com 24 meses de idade.

\section{REFERÊNCIAS}

1 Bridges P.J., Lewis P.E., Wagner W.R. \& Inskeep E.K. 1999. Follicular growth, estrus and pregnancy after fixed-time insemination in beef cows treated with intravaginal progesterone inserts and estradiol benzoate. Theriogenology. 52: 573-583.

2 Cavestany D. 1996. Estrus synchronization in lactating Holstein cows with a vaginal sponge impregnated with medroxiprogesterone acetate. In: Proceedings of the 13th International Congress on Animal Reproduction v.2. (Sydney, Australia.). pp.4-13.

3 Doray J.M., Burges J.C., Callejas S.S., Schiersmann G.C.S., Torquati O., Butler H. \& Alberio R.H. 1997. Control reproductivo en vacas de cría mediante progesterona vaginal. Efecto de varios fatores sobre la fertilidad. Archivos Medicina Veterinaria. 29: 63-68. 
4 Fike K.E., Day M.L., Inskeep E.K., Kinder J.E., Lewis P.E., Short R.E. \& Hafs H.D. 1997. Estrus and luteal function in sucled beef cows that were anestrous when treated with an intravaginal device containing progesterone with or without a subsequente injection of estradiol benzoate. Journal of Animal Science. 75: 2009-2015.

5 Gordon I. 1996. Controlled reproduction in cattle and buffaloes. London: CAB International [ Controlled Reproduction in Farm Animals Series, 1], 492p.

6 Gyawu P., Ducker M.J., Pope G.S., Saunders R.W. \& Wilson G.D.A. 1991. The value of progesterone, oestradiol benzoate and cloprostenol in controlling the timing of oestrus and ovulation in dairy cows and allowing successful fixed-time insemination. British Veterinary Journal. 147: 171-182.

7 Hanlon D.W., Williamson N.B., Wichtel J.J., Steffert I.J., Craigie A. L. \& Pfeifer D.U. 1996. The effect of estradiol benzoate administration on estrus response and synchronized pregnancy rate in dairy heifers after treatment with exogenous progesterone. Theriogenology. 45: 775-785.

8 Hanlon D.W., Williamson N.B., Wichtel J.J., Steffert I.J., Craigie A. L. \& Pfeifer D.U. 1997. Ovulatory responses and plasma luteinizing hormone concentrations in dairy heifers after treatment with exogenous progesterone and estradiol benzoate. Theriogenology. 47: 963-975.

9 Jaume C.M. \& Moraes J.C.F. 2001. Um sistema para melhorar a taxa reprodutiva em vacas de cria. Bagé: Embrapa Pecuária Sul [Série Documentos, 37]. 14p.

10 Lammoglia M.A., Short R.E., Bellows S.E., Bellows R.A., Macneil M.D. \& Hafs H.D. 1998. Induced and synchronized estrus in cattle: dose titration of estradiol benzoate in peripubertal heifers and postpartum cows after treatment with an intravaginal progesterone releasing insert and prostaglandin F2a. Journal of Animal Science. 76: 1662-1670.

11 Lane E. A., Austin E.J., Roche J.F. \& Crowe M.A. 2001. The effect of estradiol benzoate on synchrony of estrus and fertility in cattle after removal of a progesterone releasing intravaginal device. Theriogenology. 55: 1807-1818.

12 Moraes J.C.F. \& Jaume C.M. 1997. Sincrobovi: um pessário para sincronização de cios em bovinos. Revista Brasileira de Reprodução Animal. 1: 99-101.

13 Moraes J.C.F., Souza C.J.H. \& Gonçalves P.B.D. 2002. Controle do estro e da ovulação em bovinos e ovinos. In: Gonçalves P.B.D., Figueiredo J.R., Freitas V.J.F. (Eds). Biotécnicas aplicadas à reprodução animal. São Paulo: Livraria Varela, pp. 25-55.

14 Rasby R.J., Day M.L., Johnson S.K., Kinder J.E., Lynch J.M., Short R.E., Wettemann R.P. \& Hafs H.D. 1998. Luteal function and estrus in peripubertal beef heifers treated with an intravaginal progesterone releasing device with or without a subsequente injection of estradiol. Theriogenology. 50: 55-63.

15 Roche J.F. 1974. Synchronization of oestrus in heifers with implants of progesterone. Journal of Reproduction and Fertility. 41:337-344.

16 Souza C.J.H., Jaume C.M. \& Moraes J.C.F. 1999. Momento e via de suplementação de progestágeno na sincronização de cios em vacas desmamadas aos 60 dias pós-parto durante o outono. In: Anais do XIV Congresso Estadual de Medicina Veterinária ( Gramado, Brasil). p.116.

17 Wiltbank J.N., Sturges J.C., Wideman D., Lefever D.G. \& Faulkner L.C. 1971. Control of estrus and ovulation using subcutaneous implants and estrogens in beef cattle. Journal of Animal Science. 33: 600-606.

18 Xu Z.Z., Burton L.J. \& Macmillan K.L. 1996. Reproductive performance of lactating dairy cows following oestrus synchronization with progesterone, oestradiol and prostaglandin. New Zealand Veterinary Journal. 44: 99-104.

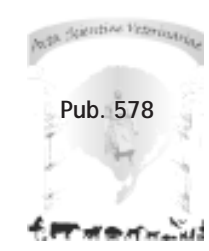

